Supporting Information

\title{
Adhesion of Bacteria to a Graphene Oxide Film
}

Jiang Ming, ${ }^{\dagger}$ Duo Sun, ${ }^{\dagger}$ Jingping Wei, Xiaolan Chen, *Nanfeng Zheng ${ }^{*}$

State Key Laboratory for Physical Chemistry of Solid Surfaces, Collaborative Innovation Center of Chemistry for Energy Materials, National \& Local Joint Engineering Research Center of Preparation Technology of Nanomaterials, College of Chemistry and Chemical Engineering,

Xiamen University, Xiamen 361005, China. E-mail: chenxl@xmu.edu.cn; nfzheng@xmu.edu.cn 


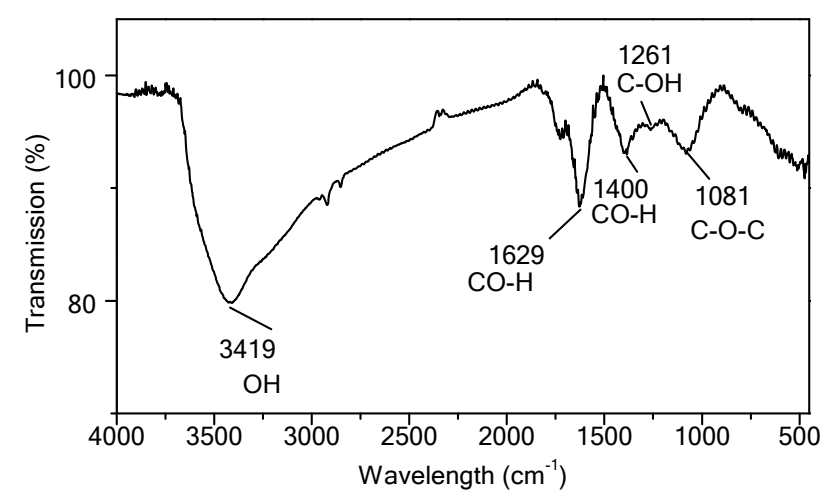

Figure S1. FTIR of GO.
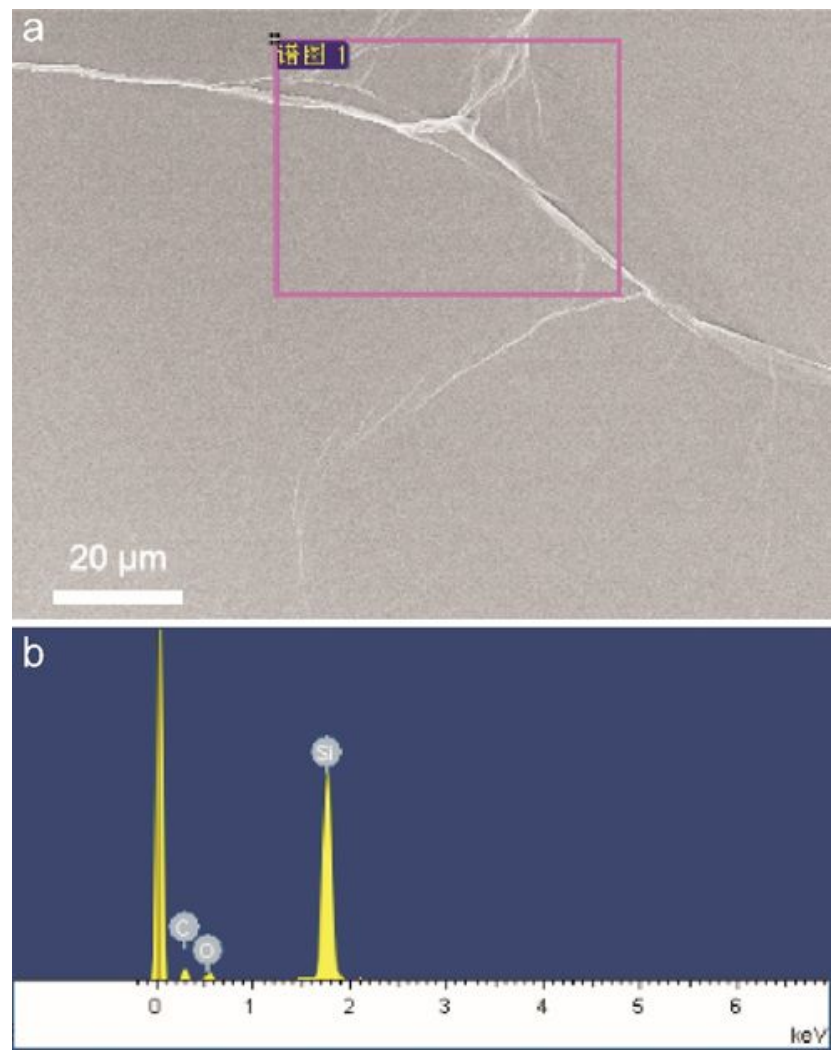

Figure S2. The energy dispersive spectrometer (EDS) was used to verify the purity of GO solution (dropped on Si substance). 


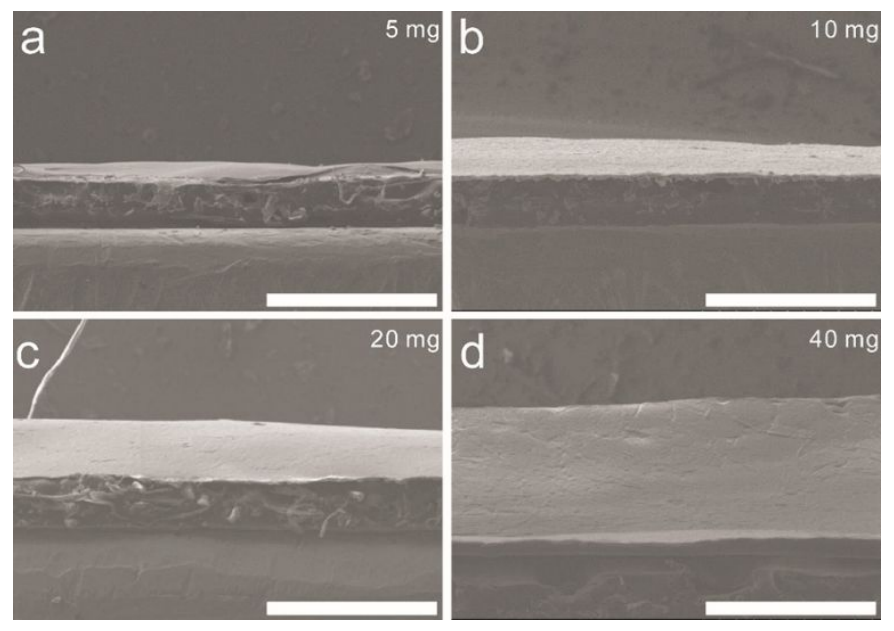

Figure S3. SEM images of the thickness for same size but different mass of GO films. Scale bar $=500$ $\mu \mathrm{m}$.

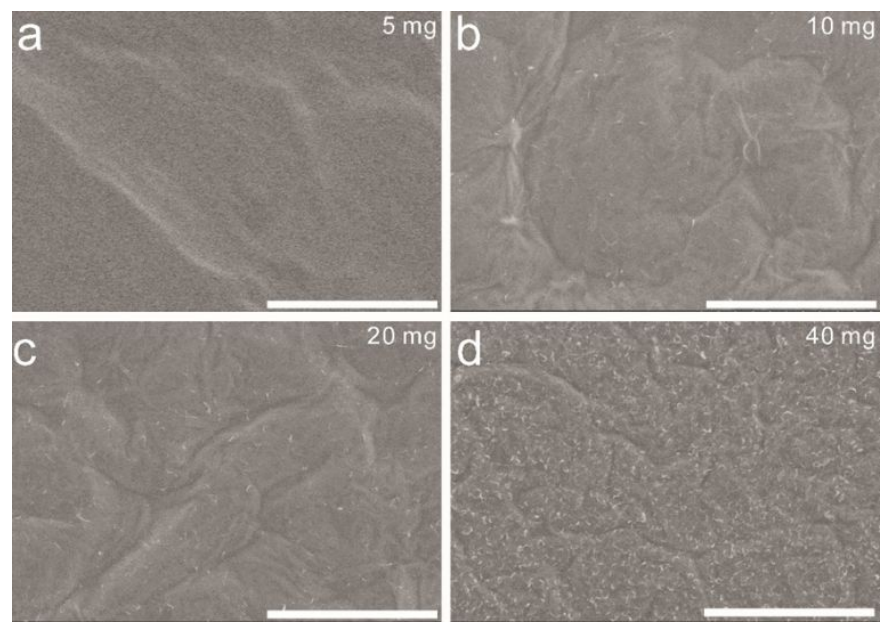

Figure S4. SEM images of GO films with different mass. Scale bar $=50 \mu \mathrm{m}$. 

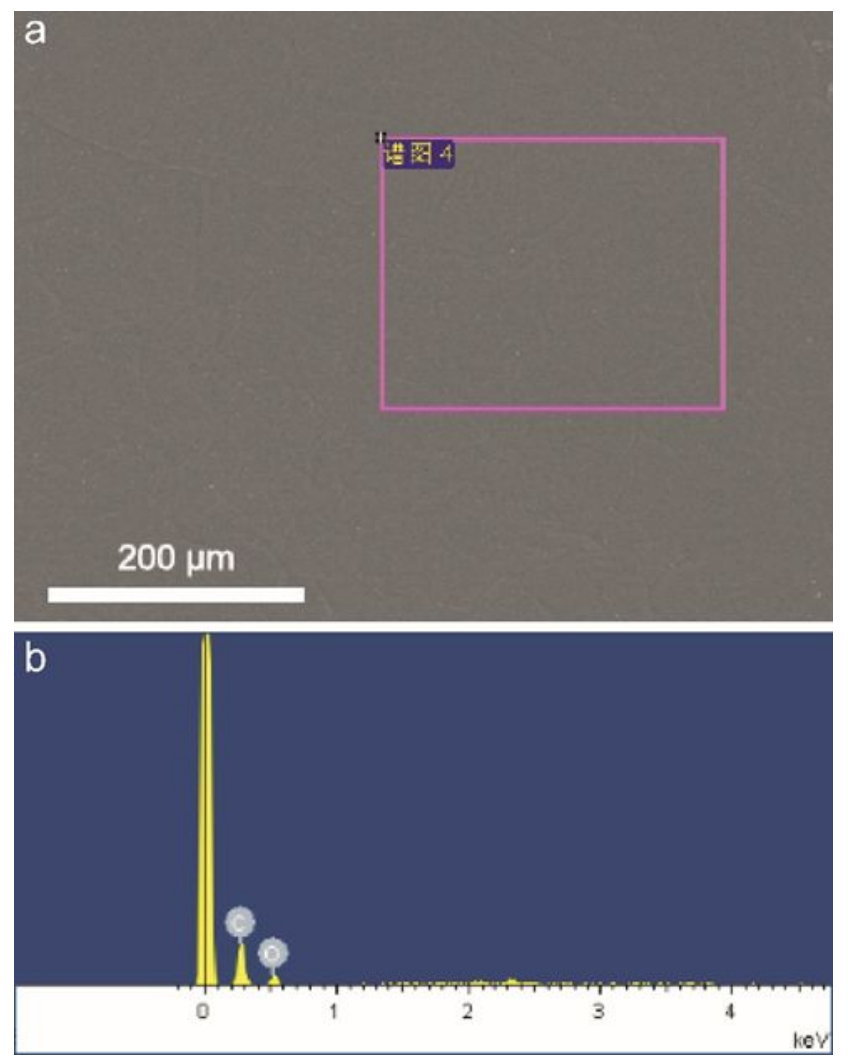

Figure S5. The energy dispersive spectrometer (EDS) was used to verify the purity of GO film.

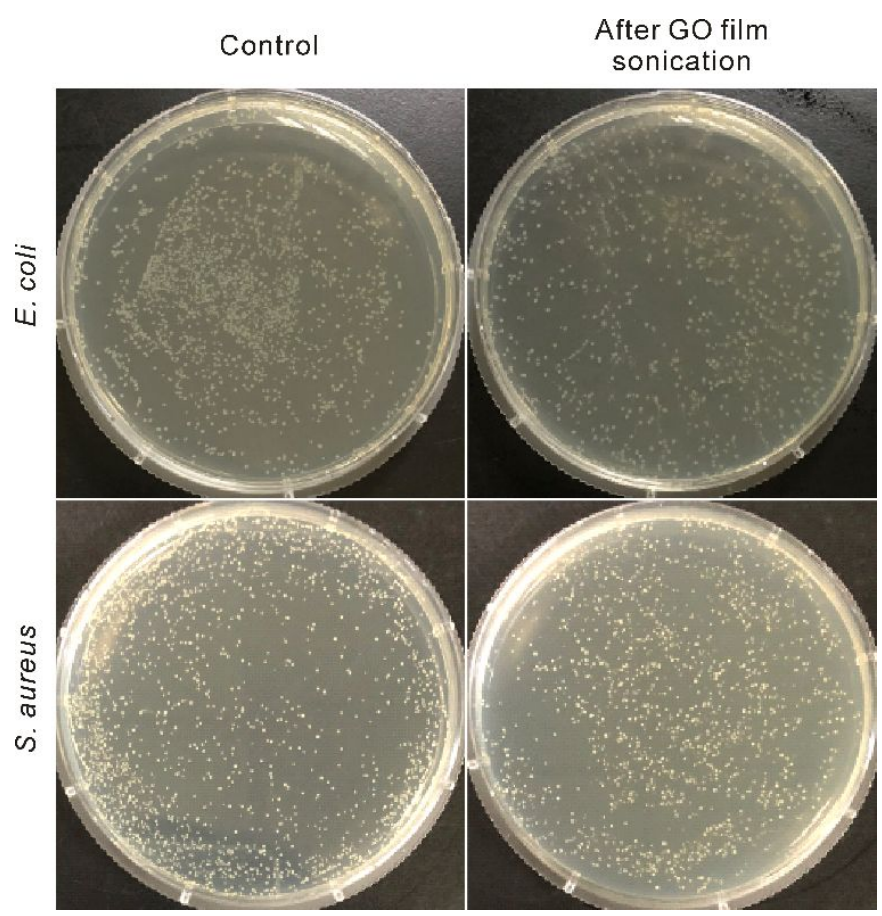

Figure S6. Viability of bacteria on GO film (E. coli and S. aureus were detached from GO films by sonication and the bacterial suspensions were then coated the agar plates). 


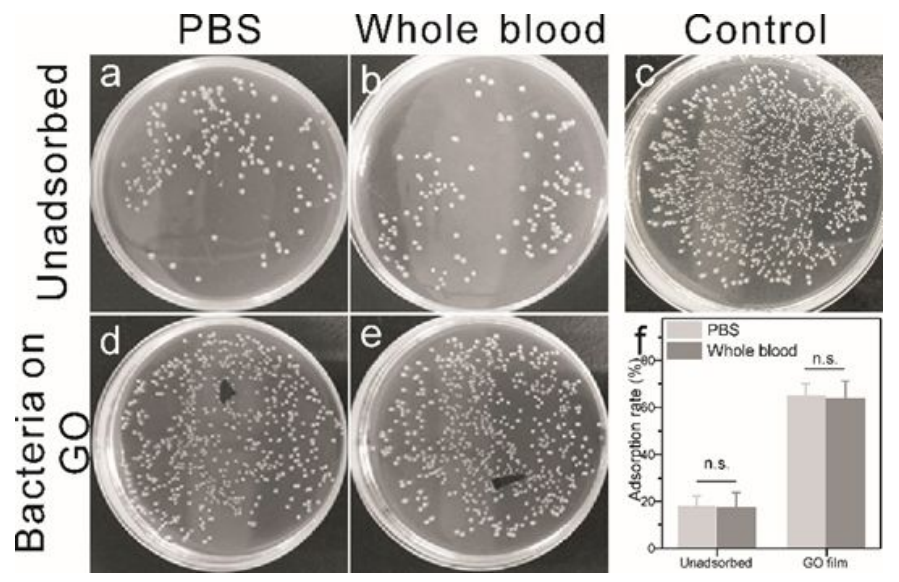

Figure S7. Adherence of $E$. coli by GO films from PBS and whole blood. (a and $b$ ) the unadhered bacteria in solution, ( $\mathrm{d}$ and e) the adhered bacteria by GO films, and (f) the histograms of adhesion rate of $E$. coli by GO films from different media ( $n=4$, n.s. means no significant difference).
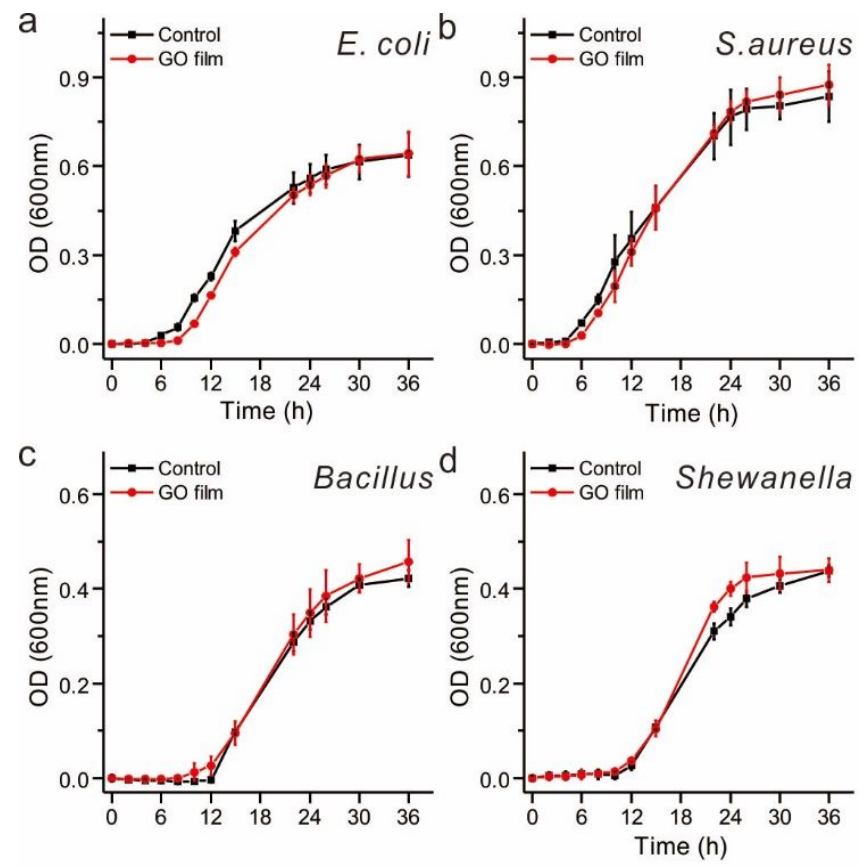

Figure S8. Growth kinetics of various bacteria after treating with PBS (control) and GO film, respectively. 


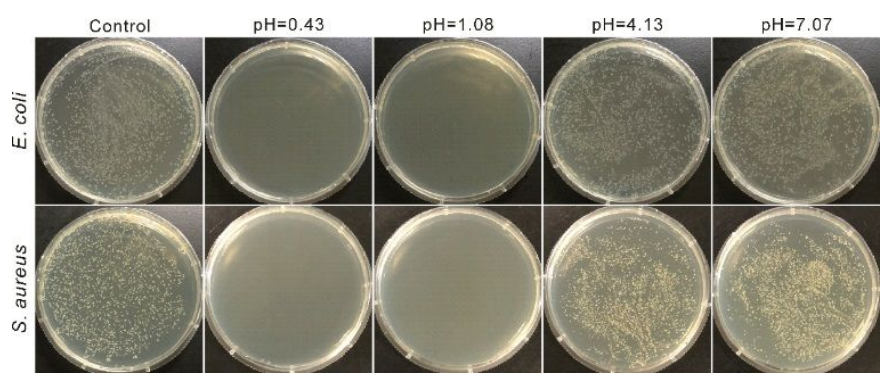

Figure S9. Influence of acidity on the antibacterial activity of GO.

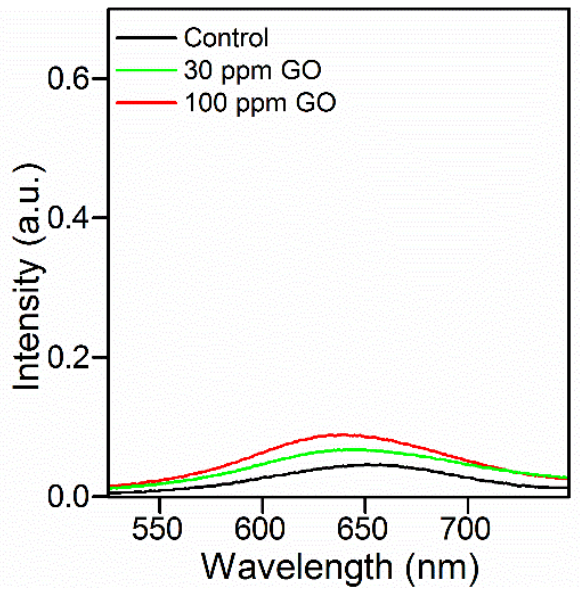

Figure S10. The peroxidase activity of GO. TMB was used as the substrate.

Table S1. The thickness of different mass of GO films

\begin{tabular}{|c|c|c|}
\hline Mass of GO film $(\mathrm{mg})$ & Thickness of GO film $(\mu \mathrm{m})$ & Standard Deviation \\
\hline 5 & 54.15 & 2.26 \\
\hline 10 & 92.42 & 7.33 \\
\hline 20 & 145.31 & 9.32 \\
\hline 40 & 339.45 & 3.32 \\
\hline
\end{tabular}




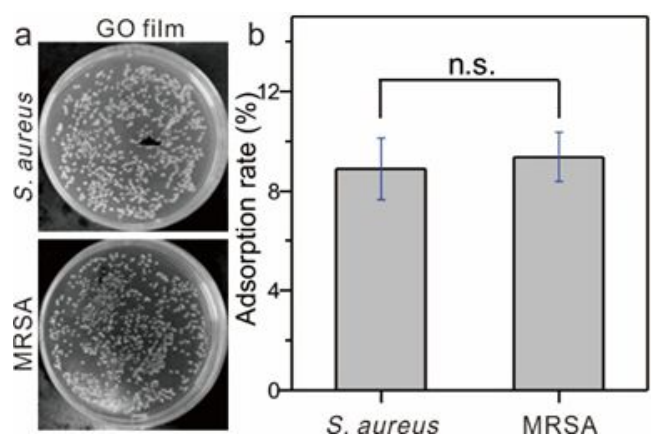

Figure S11. The adherence capability of $S$. aureus and $M R S A$ on GO films. (a) The images of agar plates with $S$. aureus and MRSA. (b) The adhere rate of $S$. aureus and MRSA. (n=4, n.s. means no significant difference).
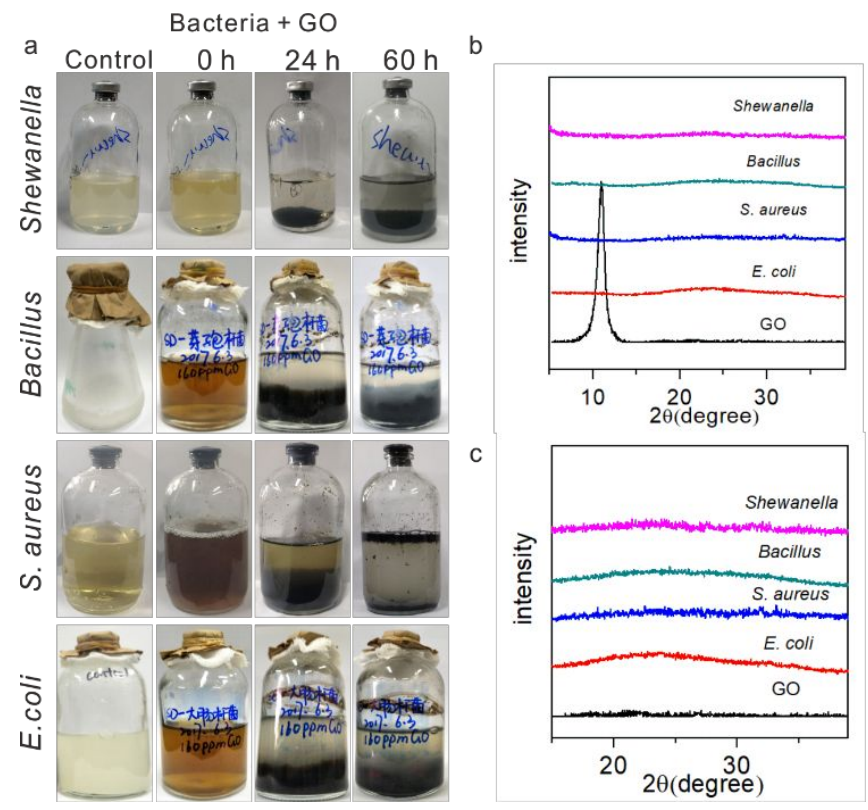

Figure S12. (a) Macroscopic pictures of GO incubated with different bacteria for $24 \mathrm{~h}$ and $60 \mathrm{~h}$, respectively. (b) XRD spectra of GO and GO-bacteria mixtures. (c) The local enlarged XRD spectra for (b).
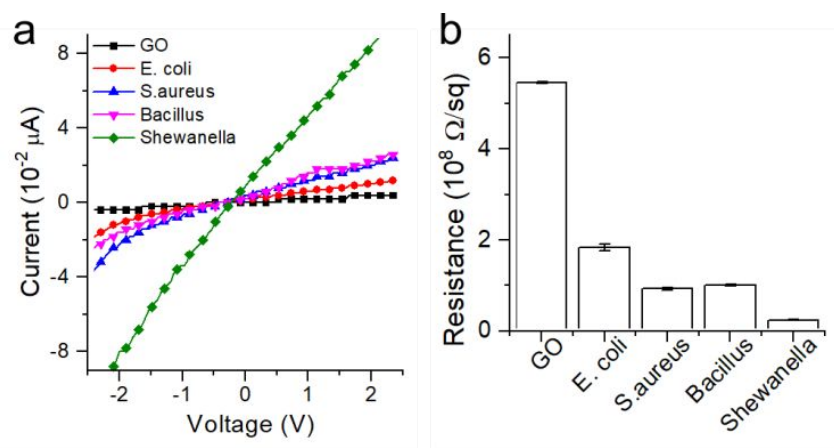
Figure S13. (a) Current-voltage diagram of the GO films after exposure to the bacterial suspensions. (b) The corresponding film resistance obtained from the I-V curves shown in (a).

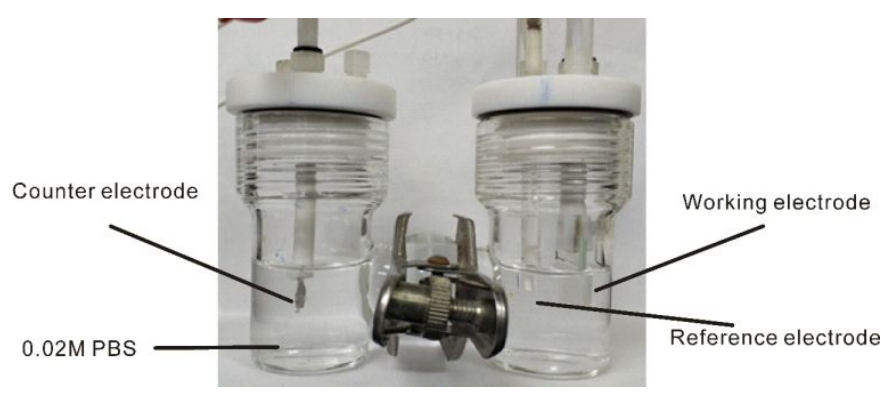

Figure S14. The picture of electrolytic tank.

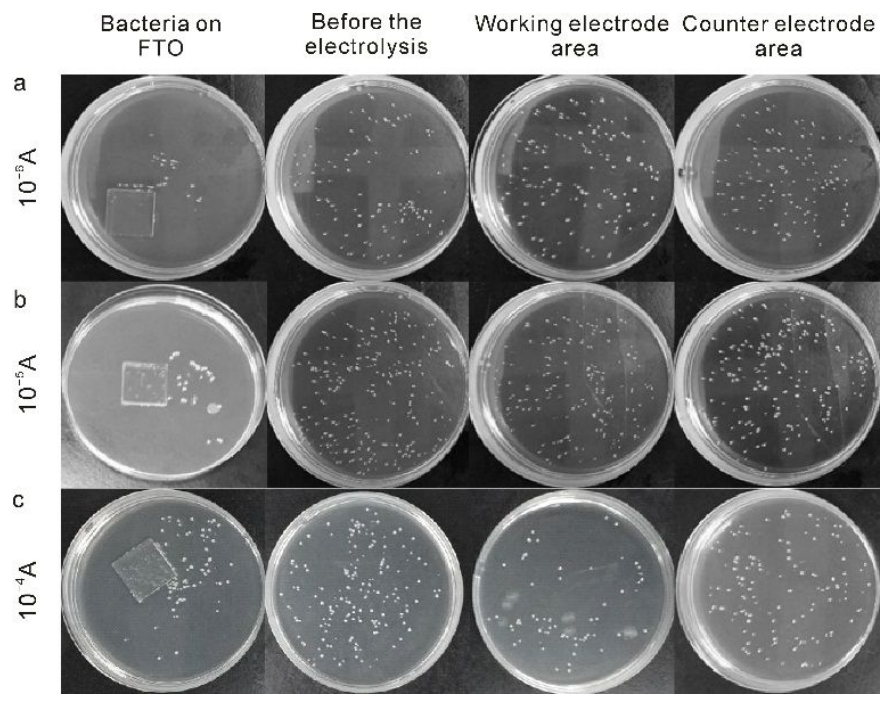

Figure S15. Influence of the impressed current on the adhesion of E. coli on FTO. Colony counting results of FTO adhered bacteria, the bacterial suspension before electrochemistry, bacterial suspensions in working electrode area and counter electrode area after $3 \mathrm{~h}$ electrolysis, respectively.

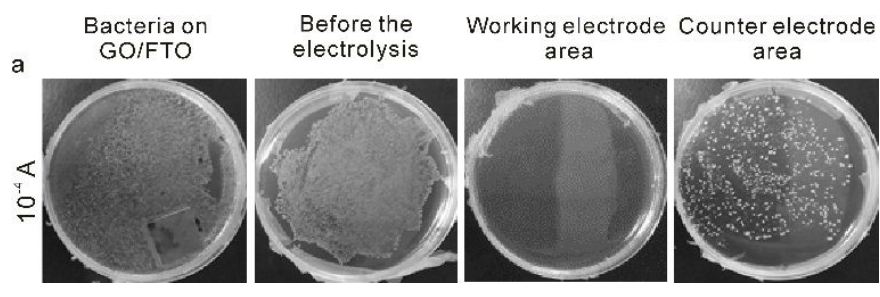

Figure S16. Influence of the impressed current on the adherence of $S$. aureus on GO/FTO. Colony counting results of GO/FTO adhered bacteria, the bacterial suspension before electrochemistry, bacterial suspensions in working electrode area and counter electrode area after $3 \mathrm{~h}$ electrolysis, respectively. 


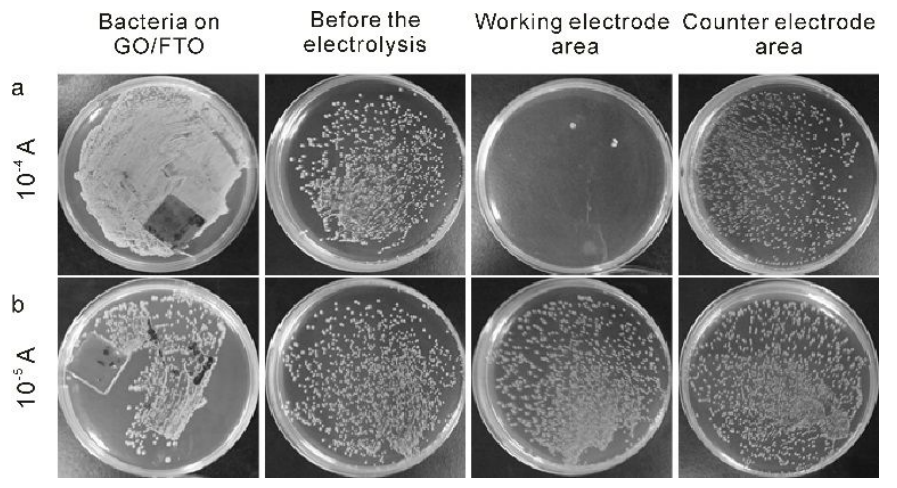

Figure S17. Influence of the impressed current on the adherence of Bacillus on GO/FTO. Colony counting results of GO/FTO adhered bacteria, the bacterial suspension before electrochemistry, bacterial suspensions in working electrode area and counter electrode area after $3 \mathrm{~h}$ electrolysis, respectively.

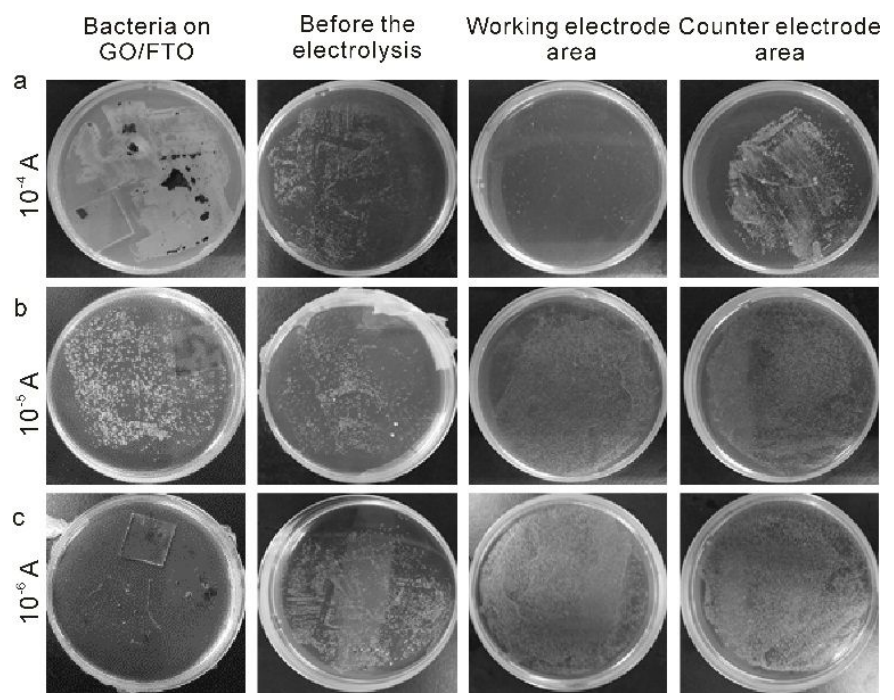

Figure S18. Influence of the impressed current on the adherence of Shewanella on GO/FTO. Colony counting results of GO/FTO adhered bacteria, the bacterial suspension before electrochemistry, bacterial suspensions in working electrode area and counter electrode area after $3 \mathrm{~h}$ electrolysis, respectively. 


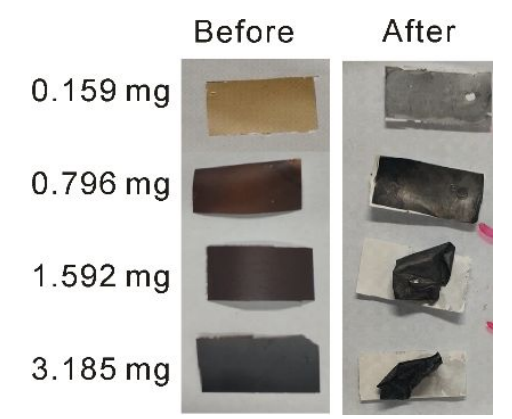

Figure S19. The GO films were reduced before and after by electrons. 